\title{
Are You an Introvert or Extrovert? Accurate Classification With Only Ten Predictors
}

\author{
Chaehan So \\ Information \& Interaction Design \\ Humanities, Arts \& Social Sciences Division, Yonsei University \\ Seoul, South Korea \\ Email: cso@yonsei.ac.kr
}

\begin{abstract}
This paper investigates how accurately the prediction of being an introvert vs. extrovert can be made with less than ten predictors. The study is based on a previous data collection of 7161 respondents of a survey on 91 personality and 3 demographic items.

The results show that it is possible to effectively reduce the size of this measurement instrument from 94 to 10 features with a performance loss of only $1 \%$, achieving an accuracy of $73.81 \%$ on unseen data. Class imbalance correction methods like SMOTE or ADASYN showed considerable improvement on the validation set but only minor performance improvement on the testing set.
\end{abstract}

Keywords-personality traits, extraversion, introversion, class imbalance, variable importance.

\section{INTRODUCTION}

In psychology, the differences between people's personality is often described on the trait level [1]. An abundance of research has provided evidence for such interindividual differences that are stable over time. One prominent example is the so-called big five model by McCrae and John [2] that postulates the existence of five independent personality traits: conscientiousness, openness, extraversion, agreeableness and neuroticism. The big five model has been confirmed by numerous validation studies, e.g. conscientiousness $(\beta=.37)$ and extraversion $(\beta=.23)$ predict subjective well-being [3], and extraversion is negatively correlated with self-consciousness $(r=-.58, p<.05)$ [4]. Many of these studies show the elevated relevance of extraversion as a predictor of many psychological phenomena.

Extraversion is often described on a bipolar continuum where low scores on extraversion are categorized as introversion [5]. Psychometrics, the science of measuring psychological dimensions, defines that personality traits can be measured as latent variables which can be captured by scales with sufficiently high internal consistency [6].

The relevance of identifying extraversion correctly becomes apparent by two complementary findings: On the one side, extraversion originates from a dopaminergic circuit involving the ventral striatum [7]. This fact may be responsible for the positive relationship between extraversion and subjective well-being consistently found in happiness research. On the other side, less extraverted people can induce positive affect by acting extraverted, i.e. by exhibiting extraverted behavior [8]. This may represent an effective measure for introverts who suffer from the negative aspects of introverted behavior like loneliness that leads to more negative affect [9].

\section{METHOD}

\section{A. Multidimensional Introversion-Extroversion Scales Dataset}

The open data movement has long entered the psychological field and led to various efforts to make interesting psychological datasets publicly available. One such offering is the Open-Source Psychometric Project [10] that collects data on a large scale on various personality assessment measurement scales. Among them, the Multidimensional Introversion-Extraversion Scales (MIES) dataset was released on 19 Aug 2019 and contains 7188 questionnaire responses.

The MIES dataset consists of the target variable $I E$, i.e. the self-identification as introvert or extrovert, and 95 features, thereof 91 items measuring the degree of extraversion on a 5point Likert scale (from $1=$ Disagree to $5=$ Agree), 3 demographic items (country, gender, English native speaker), 1 item indicating the date of survey submittal. The latter item was removed as it didn't represent any predictive value for the introvert/extrovert self-identification, leading to 94 features.

Participants were $58.1 \%$ female and $37.8 \%$ male, to a high proportion (68.9\%) English native speaker, and spread over 121 countries, mainly US (47.6\%), Great Britain (7.16\%), Canada (6.26 \%), Australia (4.50\%), Germany (2.70\%), India (2.07\%), Philippines (1.76\%), Brazil(1.27\%), Poland $(1.27 \%)$, Romania (1.08 \%), Malaysia (1.08\%), Netherlands $(1.03 \%)$, New Zealand $(0.96 \%)$, France $(0.95 \%)$ and Sweden $(0.91 \%)$.

\section{B. Technological Infrastructure}

The data analysis was performed on a virtual machine with Rstudio Server running on 96 CPU cores (Intel Skylake), and 250GB RAM in the Google Cloud Compute Engine. 


\section{Data Preprocessing}

The dataset was analyzed for missing values. 27 observations did not contain any value in the target variable ("IE" = introvert-extrovert self-identification), and thus were removed from the dataset. This removal led to a final sample size of 7161. In addition to the personality items, two technical items denoted the item position in the survey and the time elapsed for this item in milliseconds. These technical items were removed as they didn't contain any predictive information.

TABle 1: Benchmarking ACCURACy Results - Baseline Feature Set

\begin{tabular}{|c|c|c|l|}
\hline Rank & $\begin{array}{c}\text { Variable } \\
\text { Importance }\end{array}$ & Item & \multicolumn{1}{|c|}{ Item Wording } \\
\hline 1 & 100 & Q83A & I keep in the background. \\
\hline 2 & 92.412 & Q91A & I talk to a lot of different people at parties. \\
\hline 3 & 54.422 & Q82A & I don't talk a lot. \\
\hline 4 & 28.231 & Q80A & I love large parties. \\
\hline 5 & 25.257 & Q90A & I start conversations. \\
\hline 6 & 16.802 & Q81A & I am quiet around strangers. \\
\hline 7 & 14.500 & Q10A & I prefer to socialize 1 on 1, than with a group. \\
\hline 8 & 14.039 & Q84A & I don't like to draw attention to myself. \\
\hline 9 & 13.611 & Q14A & I want a huge social circle. \\
\hline 10 & 12.672 & Q7A & I spend hours alone with my hobbies. \\
\hline
\end{tabular}

Note: random forests with 10-fold cross-validation in 2 repetitions

\section{Feature Selection}

The full dataset $(n=7161)$ containing 94 features was estimated for the classification of the target variable by the random forests algorithm [11] using 10-fold cross-validation in 2 repetitions. From this estimation, the variable importance for all features were derived. Table 1 shows the relative influence on the prediction normalized by 100 for the best predictor (Q83A: "I keep in the background.").

The accuracy on the hold-out validation set was $74.49 \%$ and served as baseline for the subsequent benchmarking on the reduced dataset.

\section{E. Counterbalancing Class Imbalance}

The target variable contained three classes that were distributed unevenly. The majority class (introverted) represented $61.5 \%$ of the target variable. Two methods were applied to counterbalance the encountered class imbalance.

One of the most commonly applied methods is the synthetic minority oversampling technique (SMOTE) [12]. This method synthesizes new class instances of the minority class by an interpolation with k-nearest neighbors. The newly generated minority class samples serve to reduce the bias during training. Another class imbalance correcting method is adaptive synthesis sampling (ADASYN) [13]. This method was conceived to correct the minority class by oversampling but also adaptively give higher weights for the misclassified samples.

\section{RESULTS}

\section{A. Benchmarking on Reduced Dataset}

The dataset was substantially reduced from 94 features to the top 10 predictors of the preceding variable importance analysis. This benchmarking was run again with 10 -fold cross-validation, but with 10 instead of 2 repetitions. The dataset was split with a $85: 15$ split ratio amounting to 6087 samples in the training set and 1074 samples in the testing set.

To achieve the same data distribution, the data split was performed with stratification, maintaining the same relative frequencies for each class level. Table 2 displays the five machine learning algorithms which were selected for the benchmarking.

TABLE 2: BENCHMARKING ALGORITHMS

\begin{tabular}{|c|c|c|c|}
\hline $\mathrm{Nr}$ & Reference & Algorithm Name & $R$ Package \\
\hline 1 & {$[14]$} & k-nearest neighbors & $k n n$ \\
\hline 2 & {$[15]$} & Single-layer neural network & nnet \\
\hline 3 & {$[16]$} & Gradient boosting machines & $\mathrm{gbm}$ \\
\hline 4 & {$[11]$} & Random forests & randomForest \\
\hline 5 & {$[17]$} & Support vector machines & kernlab \\
\hline
\end{tabular}

Table 3 and Figure 1 display the results of the first benchmarking run, sorted by testing set performance. These results are interesting in the following aspects:

- The best algorithms achieve accuracy values on the cross-validation hold-out set (between 73.7 - 73.8\%) that reach approximately $99.0 \%$ of the baseline performance $(74.49 \%)$.

- This performance can be seen as a relatively good result considering it is distinctly higher than the no information rate $(61.51 \%)$.

- The accuracy between cross-validation and testing set does not differ dramatically. This result is expected due to data stratification and because cross-validation uses a hold-out set to estimate accuracy that should resemble the testing set if no tuning was performed.

- The algorithms do not differ distinctly in testing set performance, yet random forests perform weakly.

TABLE 3: BENCHMARKING RESULTS - ORIGINAL DATASET

\begin{tabular}{|c|c|c|c|c|c|}
\hline algorithm & $\begin{array}{c}\text { Cross-valid } \\
\text { Acc-mean }\end{array}$ & $\begin{array}{c}\text { Cross-valid } \\
\text { Acc-sd }\end{array}$ & $\begin{array}{c}\text { Cross-valid } \\
\text { Kappa-mean }\end{array}$ & $\begin{array}{c}\text { Cross-valid } \\
\text { Kappa-sd }\end{array}$ & $\begin{array}{c}\text { Testingset } \\
\text { Accuracy }\end{array}$ \\
\hline $\mathrm{gbm}$ & $73.83 \%$ & $1.45 \%$ & $48.80 \%$ & $2.94 \%$ & $73.81 \%$ \\
\hline $\mathrm{nnet}$ & $73.71 \%$ & $1.44 \%$ & $48.77 \%$ & $2.91 \%$ & $73.63 \%$ \\
\hline $\mathrm{knn}$ & $71.69 \%$ & $1.62 \%$ & $44.54 \%$ & $3.14 \%$ & $72.04 \%$ \\
\hline $\mathrm{rf}$ & $73.27 \%$ & $1.56 \%$ & $47.38 \%$ & $3.05 \%$ & $71.95 \%$ \\
\hline svmRadial & $73.62 \%$ & $1.56 \%$ & $47.07 \%$ & $3.31 \%$ & $71.95 \%$ \\
\hline
\end{tabular}




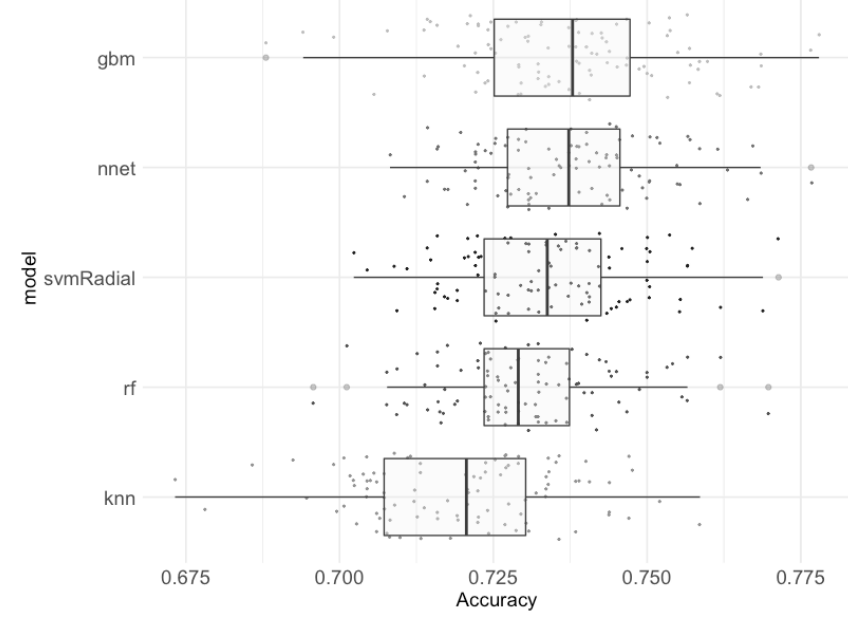

Figure 1: Benchmarking Results - Holdout Set - Original Dataset

\section{B. Benchmarking on SMOTE Dataset}

Table 4 displays the results of the second benchmarking run, performed on the dataset corrected by SMOTE.

TABLE 4: BENCHMARKING RESULTS - SMOTE DATASET

\begin{tabular}{|c|c|c|c|c|c|}
\hline algorithm & $\begin{array}{c}\text { Cross-valid } \\
\text { Acc-mean }\end{array}$ & $\begin{array}{c}\text { Cross-valid } \\
\text { Acc-sd }\end{array}$ & $\begin{array}{c}\text { Cross-valid } \\
\text { Kappa-mean }\end{array}$ & $\begin{array}{c}\text { Cross-valid } \\
\text { Kappa-sd }\end{array}$ & $\begin{array}{c}\text { Testingset } \\
\text { Accuracy }\end{array}$ \\
\hline$g b m$ & $84.23 \%$ & $1.00 \%$ & $73.60 \%$ & $1.64 \%$ & $72.79 \%$ \\
\hline rf & $84.34 \%$ & $0.81 \%$ & $73.52 \%$ & $1.37 \%$ & $72.79 \%$ \\
\hline svmRadial & $79.76 \%$ & $0.78 \%$ & $64.53 \%$ & $1.37 \%$ & $69.80 \%$ \\
\hline nnet & $78.83 \%$ & $0.92 \%$ & $63.02 \%$ & $1.61 \%$ & $68.97 \%$ \\
\hline knn & $80.43 \%$ & $0.88 \%$ & $65.86 \%$ & $1.56 \%$ & $66.08 \%$ \\
\hline
\end{tabular}

The SMOTE procedure synthesized additional samples that resulted in a total sample size of 10293. The following aspects appear to be mentionable:

- Compared to the unbalanced dataset, the results on the hold-out validation set improved substantially with SMOTE by approximately $10.8 \%$ for the best two models (gbm by $10.4 \%$ from 73.83 to $84.23 \%$, random forests by $11.1 \%$ from 73.27 to $84.34 \%$ ).

- The testing set performance for the best algorithm (gbm) decreased to a small degree of $1.02 \%$, from 73.81 to $72.89 \%$ ).

- The performance decrease on the testing set was highest for knn $(-5.96 \%)$ and nnet $(-4.66 \%)$.

\section{Benchmarking on ADASYN Dataset}

Table 5 displays the results of the second benchmarking run, performed on the dataset corrected by ADASYN.

The ADASYN procedure synthesized additional samples that resulted in a total sample size of 10501. The following comments can be made:
TABLE 5: BENCHMARKING RESULTS - ADASYN DATASET

\begin{tabular}{|c|c|c|c|c|c|}
\hline algorithm & $\begin{array}{c}\text { Cross-valid } \\
\text { Acc-mean }\end{array}$ & $\begin{array}{c}\text { Cross-valid } \\
\text { Acc-sd }\end{array}$ & $\begin{array}{c}\text { Cross-valid } \\
\text { Kappa-mean }\end{array}$ & $\begin{array}{c}\text { Cross-valid } \\
\text { Kappa-sd }\end{array}$ & $\begin{array}{c}\text { Testingset } \\
\text { Accuracy }\end{array}$ \\
\hline gbm & $84.37 \%$ & $0.92 \%$ & $73.57 \%$ & $1.50 \%$ & $73.16 \%$ \\
\hline rf & $84.62 \%$ & $0.93 \%$ & $73.75 \%$ & $1.58 \%$ & $72.51 \%$ \\
\hline svmRadial & $78.74 \%$ & $0.86 \%$ & $62.30 \%$ & $1.53 \%$ & $68.13 \%$ \\
\hline nnet & $77.19 \%$ & $0.90 \%$ & $59.41 \%$ & $1.64 \%$ & $66.82 \%$ \\
\hline knn & $79.73 \%$ & $0.79 \%$ & $64.05 \%$ & $1.43 \%$ & $65.24 \%$ \\
\hline
\end{tabular}

- Similarly to SMOTE and slightly better, the results on the hold-validation set improved substantially with ADASYN by approximately $11.0 \%$ for the best two models (gbm by $10.5 \%$ from 73.83 to $84.37 \%$, random forests by $11.4 \%$ from 73.27 to $84.62 \%$ ).

- Inversely to the validation set performance, the testing set performance for the same algorithms was slightly lower than performed on the SMOTE dataset (gbm $73.16 \%$ vs. $73.79 \%$, random forests $(72.51 \% \mathrm{vs}$. $72.79 \%)$.

- The standard deviation on the validation set metrics (accuracy and kappa) was slightly higher on the ADASYN dataset than on the SMOTE dataset.

\section{Variable Importance Analysis}

The tree-based algorithm implementations, random forests and $\mathrm{gbm}$, provide a ranking of variable importance, i.e. a ranking of all features according to their relative influence for the prediction of the target value. The following compares the variable importance between these two algorithms.

Figure 2 shows the visualization of the variable importance for the gbm algorithm. The ranking is largely consistent with the initial ranking when all 91 items were considered. However, the importance values differ, e.g. Q84A has an importance value of 0 whereas it scored 13.6 before.

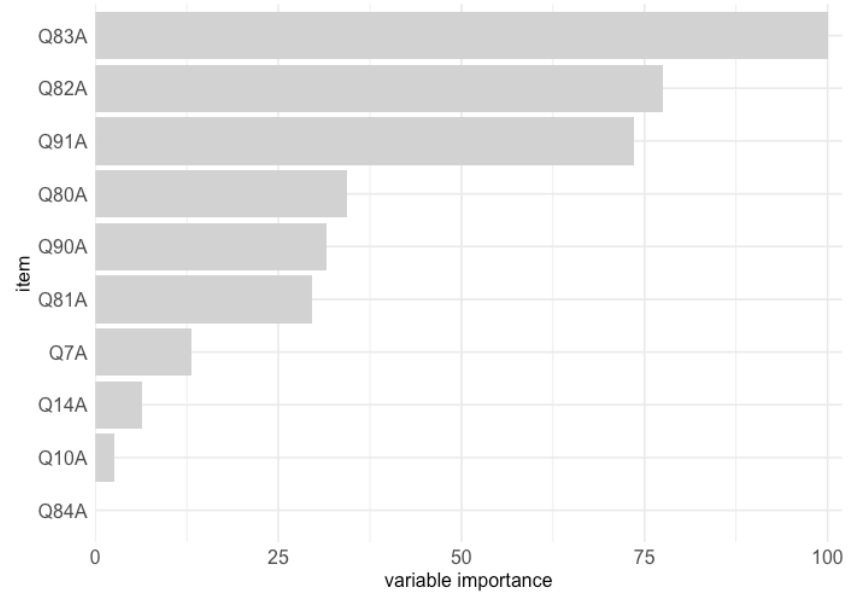

Figure 2: Variable Importance - GBM 
The variable importance of random forests shows a completely different picture. As can be seen in Figure 3, random forests acknowledge all ten features in a small range between $75-100$.

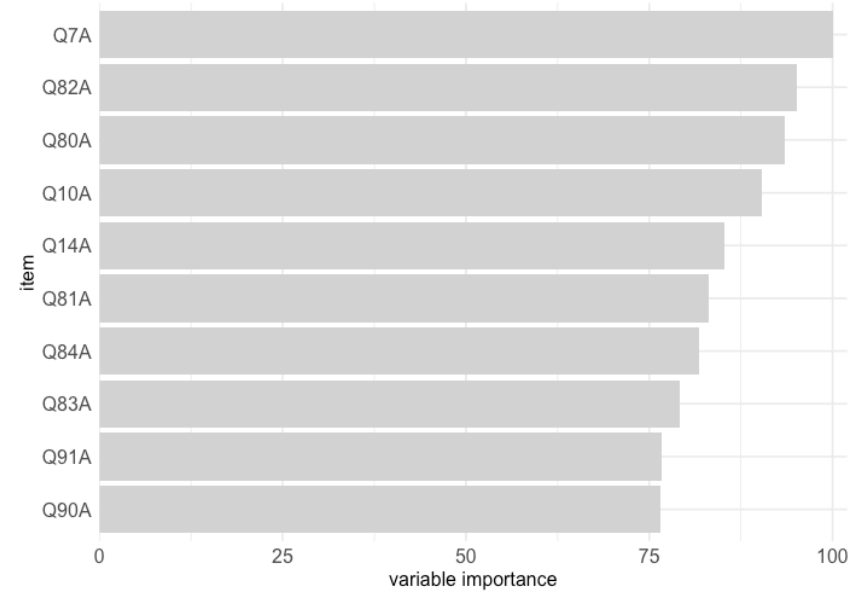

Figure 3: Variable Importance - random forests

This drastic difference illustrates the completely different mechanism between the two algorithms. Random forests grow a high number of decision trees and average their results by majority vote. The frequent reuse of all features (bagging) ensures a representative estimation of their importance independent from random partitioning of the bags. In contrast, gradient boosting machines iteratively grow a single tree and include the error into the next model, boosting the misclassifications in the next iteration. This has proved very efficient but is somewhat more prone to the random selection at the beginning iteration of the algorithm.

\section{DISCUSSION}

The current study contributes to the literature in three ways. First, it predicted people's self-identification as introvert or extrovert by a relatively high accuracy of $73.8 \%$ on unseen data. Second, it reduced a measurement instrument from 94 to only 10 features reaching approximately $99 \%$ of the accuracy achieved with the full dataset. Third, it applied and compared two class imbalance correction methods, finding ADASYN yielding slightly better results than SMOTE, with a minimal improvement on testing set performance. Fourth, it compared the variable importance ranking between $\mathrm{gbm}$ and random forests revealing the difference of the underlying algorithm principles.

\section{ACKNOWLEDGMENT}

This research was supported by the Yonsei University Faculty Research Fund of 2019-22-0199.

\section{REFERENCES}

[1] P. G. Zimbardo, "Personality: Theories of the Whole Person," in Psychology Core Concepts, 2012, pp. 412-457.

[2] R. R. McCrae and O. P. John, "An introduction to the five-factor model and its applications," J. Pers., vol. 60, no. 2, pp. 175-215, 1992.

[3] A. Weiss, T. C. Bates, and M. Luciano, "Happiness Is a Personal(ity) Thing: The Genetics of Personality and Well-Being in a Representative Sample," Psychol. Sci., vol. 19, no. 3, pp. 2052010, 2008.

[4] N. I. Eisenberger, M. D. Lieberman, and A. B. Satpute, "Personality from a controlled processing perspective: an fMRI study of neuroticism, extraversion, and self-consciousness.," Cogn. Affect. Behav. Neurosci., vol. 5, no. 2, pp. 169-181, 2005.

[5] J. Wilt and W. Revelle, "Extraversion," in Handbook of Individual Differences in Social Behavior, M. Leary and R. Hoyle, Eds. New York: Guilford, 2009, pp. 27-45.

[6] B. G. Tabachnick and L. S. Fidell, Using Multivariate Statistics. Boston: Pearson, 2019.

[7] C. A. Hutcherson, P. R. Goldin, W. Ramel, K. McRae, and J. J. Gross, "Attention and emotion influence the relationship between extraversion and neural response," Soc. Cogn. Affect. Neurosci., vol. 3, no. 1, pp. 71-79, 2008.

[8] W. Fleeson, A. B. Malanos, and N. M. Achille, "An intraindividual process approach to the relationship between extraversion and positive affect: Is acting extraverted as 'good' as being extraverted?," J. Pers. Soc. Psychol., vol. 83, no. 6, pp. 14091422, 2002.

[9] D. H. Saklofske and R. A. Yackulic, "Personality predictors of loneliness," Pers. Individ. Dif., vol. 10, no. 4, pp. 467-472, 1989.

[10] "Open-Source Psychometric Project." .

[11] A. Liaw and M. Wiener, "Classification and Regression by randomForest," $R$ News, vol. 2, no. 3, pp. 18-22, 2002.

[12] K. W. P. Chawla, N.V., Bowyer, K.W., Hall, L.O., "SMOTE: Synthetic Minority Over-Sampling Technique. Journal of Artificial Intelligence Research," J. Artif. Intell. Res., vol. 16, pp. 321-357, 2002.

[13] H. He, Y. Bai, E. A. Garcia, and S. Li, "ADASYN: Adaptive synthetic sampling approach for imbalanced learning," Proc. Int. Jt. Conf. Neural Networks, no. 3, pp. 1322-1328, 2008.

[14] M. Kuhn, "caret: Classification and Regression Training.” 2018.

[15] W. N. Venables and B. D. Ripley, Modern Applied Statistics with S, Fourth. New York: Springer, 2002.

[16] B. Greenwell, B. Boehmke, J. Cunningham, and G. B. M. Developers, "gbm: Generalized Boosted Regression Models." 2019.

[17] A. Karatzoglou, A. Smola, K. Hornik, and A. Zeileis, "kernlab -- An S4 Package for Kernel Methods in R," J. Stat. Softw., vol. 11, no. 9, pp. 1-20, 2004. 